This item was submitted to Loughborough's Research Repository by the author.

Items in Figshare are protected by copyright, with all rights reserved, unless otherwise indicated.

\title{
Bound states in coupled guides. II. Three dimensions.
}

PLEASE CITE THE PUBLISHED VERSION

LICENCE

CC BY-NC-ND 4.0

\section{REPOSITORY RECORD}

Linton, C.M., and Keith Ratcliffe. 2019. "Bound States in Coupled Guides. II. Three Dimensions.”. figshare. https://hdl.handle.net/2134/306. 


\title{
Bound states in coupled guides. II. Three dimensions. C. M. Linton and K. Ratcliffe ${ }^{1}$
}

\begin{abstract}
We compute bound-state energies in two three-dimensional coupled waveguides, each obtained from the two-dimensional configuration considered in part I by rotating the geometry about a different axis. The first geometry consists of two concentric circular cylindrical waveguides coupled by a finite length gap along the axis of the inner cylinder and the second is a pair of planar layers coupled laterally by a circular hole. We have also extended the theory for this latter case to include the possibility of multiple circular windows. Both problems are formulated using a mode-matching technique, and in the cylindrical guide case the same residue calculus theory as used in I is employed to find the bound-state energies. For the coupled planar layers we proceed differently, computing the zeros of a matrix derived from the matching analysis directly.
\end{abstract}

\footnotetext{
${ }^{1}$ Department of Mathematical Sciences, Loughborough University, LE11 3TU, UK (c.m.linton@lboro.ac.uk).
} 


\section{Introduction}

Here we extend the ideas from part I to three dimensions and consider two distinct types of problem. First we treat the case where the two-dimensional laterally coupled waveguide discussed in I is rotated about the $x$-axis to produce a three-dimensional waveguide. The guide then consists of pair of concentric circular cylindrical guides of widths $b$ and $d$ coupled by a finite gap in the inner cylinder. We seek bound states whose energies are below the first cut-off for wave propagation down the guide using the residue calculus technique. Secondly, we consider the case where the coupled waveguide considered in I is rotated about the $y$-axis. The waveguide consists of two planar layers of widths $b$ and $d-b$ coupled through a circular hole of radius $a$ in the common boundary. In fact we set up the problem for an arbitrary number of arbitrarily sized circular windows.

Early work on acoustic resonances in circular cylindrical waveguides was done by Ursell (1991), who considered a rigid cylinder with a rigid sphere placed on the axis. Using the method of multipole expansions Ursell was able to prove the existence of resonant states with certain angular variation, provided the sphere was sufficiently small. The method presented in this paper is similar to that used by Evans and Linton (1994), who developed an approximate solution for the existence of trapped modes in an infinitely long, rigid, circular cylindrical tube containing a concentric, rigid, open-ended circular cylinder of finite length. Linton and McIver (1998) proved that acoustic resonances can exist when any rigid, thin obstacle is placed in a rigid cylindrical waveguide of constant cross-section in such a way that its normal is everywhere perpendicular to the generators of the cylinder. Similar results were also given in Groves (1998) and Davies and Parnovski (1998). The example of a cylindrical sleeve inside a circular cylindrical waveguide with Neumann conditions on all boundaries considered in Evans and Linton (1994) was recalculated using the residue calculus technique and extended to cover different angular variations.

In all the above examples, Neumann boundary conditions were imposed on the cylindrical waveguide. A cylindrical guide with Dirichlet conditions on the boundary was considered by Witsch (1990). Witsch used the same idea as Ursell in that he specified the angular variation to produce a cut-off and then used a minimum-maximum principle to provide examples of eigenvalues that can occur in the waveguide.

Much less work has been done on the second problem. Exner and Vugalter (1997) considered the case when the hole was sufficiently small so that only one eigenvalue occured below the continuous spectrum. The authors were then able to provide upper and lower asymptotic bounds on the gap between the eigenvalue and the continuous spectrum. Further asymptotic results were derived in Popov (2002).

\section{Coupled cylindrical guides}

In this section we consider a rotation of the waveguide used in I about the $x$-axis. We introduce cylindrical polar coordinates $(r, \theta, x)$ so that the outer surface of the guide is at 


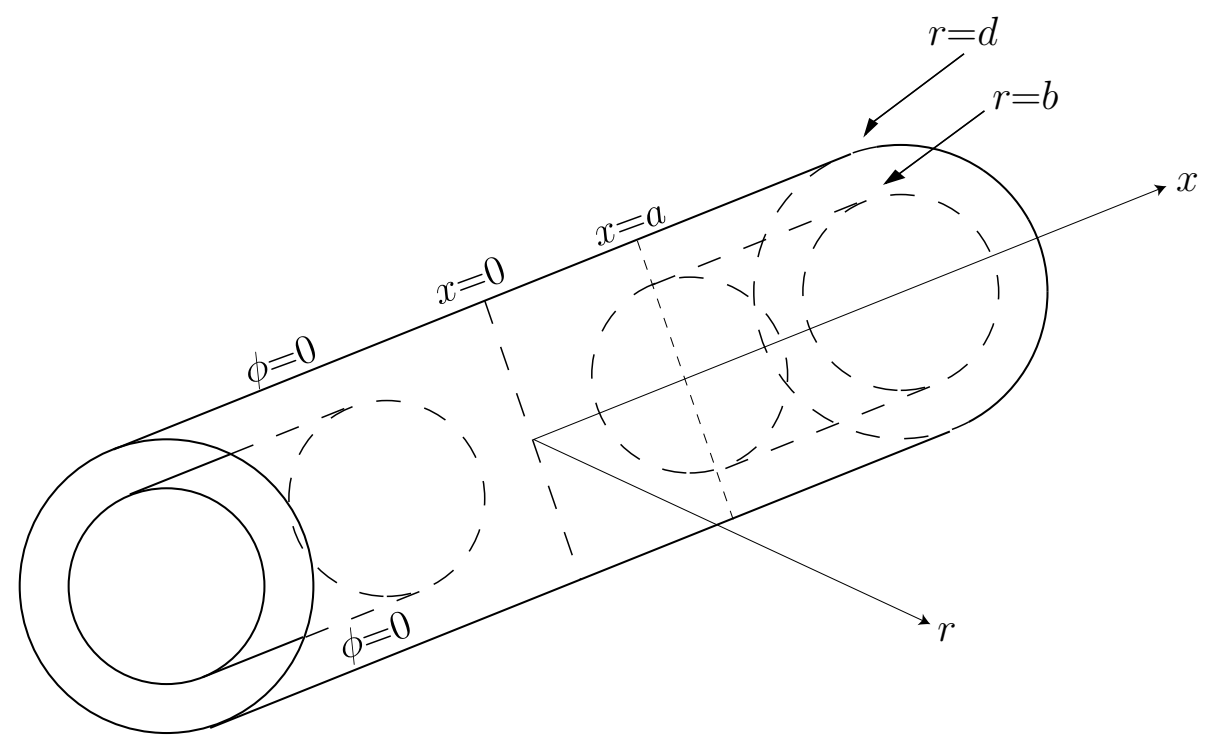

Figure 1: Definition sketch.

$r=d$. Inside the guide is placed an infinite, concentric cylinder of radius $b(<d)$ which has a gap of length $2 a$ along its axis. The inner cylinder is placed so that its surface is at $r=b$ and the gap is at $-a<x<a$, as shown in Figure 1. The resulting geometry is axisymmetric about the line $r=0$, hence we are able to look for modes with angular variation $\cos m \theta$, where $m \in \mathbb{N}_{0}$. The quantity $m$ is to be regarded as fixed in what follows.

The geometry is symmetric about $x=0$, allowing us only to consider the region $x>0$ and seek modes which are either symmetric or antisymmetric about $x=0$. We begin by seeking modes symmetric about $x=0$ by looking for non-trivial solutions $\phi(r, \theta, x)$, which satisfy

$$
\frac{\partial \phi}{\partial x}=0 \text { on } x=0,0<r<d .
$$

The function $\phi(r, \theta, x)$ must also satisfy the Helmholtz equation within the waveguide,

$$
\left(\nabla^{2}+k^{2}\right) \phi=0, \quad 0<r<d, x>0 \text { except on } r=b, x>a,
$$

and is subject to Dirichlet boundary conditions on the cylinders

$$
\begin{aligned}
& \phi=0 \text { on } r=b, x>a, \\
& \phi=0 \text { on } r=d, x>0,
\end{aligned}
$$

and a radiation condition that stops waves propagating to infinity,

$$
\phi \rightarrow 0 \text { as } x \rightarrow \infty \text {. }
$$

Finally we assume that $\phi$ is non-singular and

$$
\frac{\partial \phi}{\partial \rho}=O\left(\rho^{-\frac{1}{2}}\right) \text { as } \rho=\left[(x-a)^{2}+(r-b)^{2}\right]^{\frac{1}{2}} \rightarrow 0,
$$


anticipating singular behaviour in the derivative of $\phi$ at the edge. The changes resulting from replacing (2.1) by an antisymmetric condition will be discussed later.

As in the two-dimensional case considered in I, we divide the interior of the guide into three parts. Region $I$ is the annular region between the outer and inner cylinders, i.e. $\{r, \theta, x: b<r<d, x>a\}$, region $I I$ is the interior of the inner cylinder, i.e. $\{r, \theta, x$ : $0<r<b, x>a\}$, and region $I I I$ is the gap of the inner cylinder, i.e. $\{r, \theta, x: 0<r<$ $d, 0<x<a\}$. We can represent the function $\phi$ by a function $\phi_{i}(r, \theta, x)=\hat{\phi}_{i}(r, x) \cos m \theta$, $\left(i=1,2,3, m \in \mathbb{N}_{0}\right)$ in each region and apply the following continuity conditions at each region's boundary

$$
\hat{\phi}_{i}=\hat{\phi}_{3}, \quad \frac{\partial \hat{\phi}_{i}}{\partial x}=\frac{\partial \hat{\phi}_{3}}{\partial x}, \quad \text { on } L_{i}, i=1,2,
$$

where $L_{1}$ is $x=a, b<r<d, L_{2}$ is $x=a, 0<r<b$ and we write $L_{3}=L_{1} \cup L_{2}$.

Complete orthogonal sets of functions of $r$ in each of the three regions are defined in terms of Bessel functions as follows. Let $\nu_{m n}$ be the $n$th positive zero of the cross-product $J_{m}(\eta d) Y_{m}(\eta b)-Y_{m}(\eta d) J_{m}(\eta b)$, then functions appropriate for region $I$ are

$$
\Psi_{m n}^{(1)}(r)=p_{m n}^{(1)}\left[J_{m}\left(\nu_{m n} r\right) Y_{m}\left(\nu_{m n} b\right)-Y_{m}\left(\nu_{m n} r\right) J_{m}\left(\nu_{m n} b\right)\right], \quad n \in \mathbb{N} .
$$

This function satisfies (2.3) and (2.4), and with

$$
p_{m n}^{(1)}=\frac{\nu_{m n} \pi^{\frac{1}{2}}}{2^{\frac{1}{2}}}\left(\frac{J_{m}^{2}\left(\nu_{m n} b\right)}{J_{m}^{2}\left(\nu_{m n} d\right)}-1\right)^{-\frac{1}{2}},
$$

we have the orthogonality condition (see Jones 1986, p. 228)

$$
\int_{L_{i}} r \Psi_{m n}^{(i)}(r) \Psi_{m s}^{(i)}(r) \mathrm{d} r=\delta_{n s}, \quad n, s \in \mathbb{N},
$$

with $i=1$, where $\delta_{n s}$ is the Kronecker delta.

Similarly, for region $I I$ we let $j_{m n}$ be the $n$th non-negative zero of $J_{m}(\eta)$ and define $\mu_{m n} b=j_{m n}$. We then define

$$
\Psi_{m n}^{(2)}(r)=p_{m n}^{(2)} J_{m}\left(\mu_{m n} r\right), \quad n \in \mathbb{N}
$$

where

$$
p_{m n}^{(2)}=\frac{2^{\frac{1}{2}}}{b J_{m+1}\left(\mu_{m n} b\right)},
$$

which satisfies (2.3) and the orthogonality condition (2.10) with $i=2$.

For region $I I I$ we let $\lambda_{m n} d=j_{m, n+1}$ and define

$$
\Psi_{m n}^{(3)}(r)=p_{m n}^{(3)} J_{m}\left(\lambda_{m n} r\right) \quad n \in \mathbb{N}_{0}
$$

where

$$
p_{m n}^{(3)}=\frac{2^{\frac{1}{2}}}{d J_{m+1}\left(\lambda_{m n} d\right)} .
$$


Then $\Psi_{m n}^{(3)}$ satisfies (2.4) and the orthogonality condition (2.10) with $i=3$.

The appropriate eigenfunction expansions for $\hat{\phi}$ are now

$$
\begin{array}{ll}
\hat{\phi}_{1}(r, x)=\sum_{n=1}^{\infty} U_{m n}^{(1)} \frac{\mathrm{e}^{-\alpha_{m n}(x-a)}}{-\alpha_{m n}} \Psi_{m n}^{(1)}(r), & \alpha_{m n}=\left(\nu_{m n}^{2}-k^{2}\right)^{\frac{1}{2}}, \\
\hat{\phi}_{2}(r, x)=\sum_{n=1}^{\infty} U_{m n}^{(2)} \frac{\mathrm{e}^{-\beta_{m n}(x-a)}}{-\beta_{m n}} \Psi_{m n}^{(2)}(r), & \beta_{m n}=\left(\mu_{m n}^{2}-k^{2}\right)^{\frac{1}{2}}, \\
\hat{\phi}_{3}(r, x)=\sum_{n=0}^{\infty} U_{m n}^{(3)} \frac{\cosh \gamma_{m n} x}{\gamma_{m n} \sinh \gamma_{m n} a} \Psi_{m n}^{(3)}(r), & \gamma_{m n}=\left(\lambda_{m n}^{2}-k^{2}\right)^{\frac{1}{2}} .
\end{array}
$$

For decay down the guide we require $\alpha_{m n}$ and $\beta_{m n}$ to be real and positive for all $n$ and so we must have

$$
k d<\nu_{m 1} d \quad \text { and } \quad k d<\mu_{m 1} d .
$$

So as to allow one wave-like mode in the inner region, we also require $\gamma_{m 0}$ to be purely imaginary, but $\gamma_{m n}$ to be real and positive for all other values of $n$, and hence

$$
k d>\lambda_{m 0} d=j_{m 1} .
$$

We therefore anticipate that a necessary condition for the existence of bound states is

$$
j_{m 1}<k d<\min \left(\mu_{m 1} d, \nu_{m 1} d\right) .
$$

\section{Bound states below the first cut-off}

We now use the continuity conditions (2.7) and proceed as in I. We obtain

$$
\begin{array}{ccc}
U_{m s}^{(3)} & =\sum_{n=1}^{\infty} U_{m n}^{(1)} d_{n s}+\sum_{n=1}^{\infty} U_{m n}^{(2)} e_{n s}, & s \in \mathbb{N}_{0}, \\
U_{m s}^{(3)} \frac{\operatorname{coth} \gamma_{m s} a}{\gamma_{m s}}=\sum_{n=1}^{\infty} \frac{U_{m n}^{(1)}}{-\alpha_{m n}} d_{n s}+\sum_{n=1}^{\infty} \frac{U_{m n}^{(2)}}{-\beta_{m n}} e_{n s}, & s \in \mathbb{N}_{0},
\end{array}
$$

where we have defined

$$
\begin{aligned}
d_{n s} & =\frac{1}{d} \int_{L_{1}} r \Psi_{m n}^{(1)}(r) \Psi_{m s}^{(3)}(r) \mathrm{d} r \\
e_{n s} & =\frac{1}{d} \int_{L_{2}} r \Psi_{m n}^{(2)}(r) \Psi_{m s}^{(3)}(r) \mathrm{d} r .
\end{aligned}
$$

Using various standard integrals, recurrence relations, and Wronskian relations for Bessel functions, we can show that provided $\nu_{m n} \neq \lambda_{m s}$ and $\mu_{m n} \neq \lambda_{m s}$,

$$
\begin{array}{ll}
d_{n s}=-\frac{2 \nu_{m n} J_{m}\left(\lambda_{m s} b\right)}{\left(\nu_{m n}^{2}-\lambda_{m s}^{2}\right) \pi^{\frac{1}{2}} d J_{m+1} \lambda_{m s} d}\left(\frac{J_{m}^{2}\left(\nu_{m n} b\right)}{J_{m}^{2}\left(\nu_{m n} d\right)}-1\right)^{-\frac{1}{2}}, & s \in \mathbb{N}_{0}, n \in \mathbb{N}, \\
e_{n s}=\frac{2 \mu_{m n} J_{m}\left(\lambda_{m s} b\right)}{d J_{m+1}\left(\lambda_{m s} d\right)\left(\mu_{m n}^{2}-\lambda_{m s}^{2}\right)}, & s \in \mathbb{N}_{0}, n \in \mathbb{N} .
\end{array}
$$


Eliminating $U_{m n}^{(3)}$ from (2.21) and (2.22) and using (2.25) and (2.26), we obtain after some simplification

$$
\sum_{n=1}^{\infty} U_{m n}\left(\frac{1}{\alpha_{m n}-\gamma_{m s}}+\frac{\zeta_{m s}}{\alpha_{m n}+\gamma_{m s}}\right)-\sum_{n=1}^{\infty} V_{m n}\left(\frac{1}{\beta_{m n}-\gamma_{m s}}+\frac{\zeta_{m s}}{\beta_{m n}+\gamma_{m s}}\right)=0,
$$

where $s \in \mathbb{N}_{0}$ and we have defined

$$
U_{m n}=\frac{U_{m n}^{(1)} \nu_{m n}}{\pi^{\frac{1}{2}} \alpha_{m n}}\left(\frac{J_{m}^{2}\left(\nu_{m n} b\right)}{J_{m}^{2}\left(\nu_{m n} d\right)}-1\right)^{-\frac{1}{2}}, \quad V_{m n}=\frac{U_{m n}^{(2)} \mu_{m n}}{\beta_{m n}}, \text { and } \zeta_{m s}=\mathrm{e}^{-2 \gamma_{m s} a} .
$$

The singular behaviour required by condition (2.6) again influences the asymptotic behaviour of $U_{m n}$ and $V_{m n}$ as $n \rightarrow \infty$. As in I we can show that the edge condition is satisfied if both $U_{m n}=O\left(n^{-\frac{1}{2}}\right)$ and $V_{m n}=O\left(n^{-\frac{1}{2}}\right)$ as $n \rightarrow \infty$.

We now use the residue calculus technique of Mittra and Lee (1971), exactly as in I; the details are different but the procedure is essentially the same as before. We will make just one remark. The standard infinite product representation of the gamma function can be used to show that

$$
\prod_{n=1}^{\infty} \frac{\left(1-\frac{z d}{(n+m / 2+3 / 4) \pi}\right)}{\left(1-\frac{z c}{n \pi}\right)\left(1-\frac{z b}{(n+m / 2-1 / 4) \pi}\right)}=\frac{\Gamma(m / 2+7 / 4) \Gamma(1-z c / \pi) \Gamma(m / 2+3 / 4-z b / \pi)}{\Gamma(m / 2+7 / 4-z d / \pi) \Gamma(m / 2+3 / 4)}
$$

and then the asymptotic forms (see Abramowitz and Stegun 1965, eqns 9.5.12 and 9.5.27)

$$
\begin{aligned}
\lambda_{m n} d=j_{m, n+1} & \sim(n+m / 2+3 / 4) \pi+O(1 / n), \\
\mu_{m n} b=j_{m n} & \sim(n+m / 2-1 / 4) \pi+O(1 / n), \\
\nu_{m n}(d-b) & \sim n \pi+O(1 / n)
\end{aligned}
$$

as $n \rightarrow \infty$ can be used together with Stirling's formula to derive the necessary asymptotic form for the function $f(z)$ equivalent to (3.18) of I.

The condition for the existence of bound states turns out to be

$$
\gamma_{m}^{\prime}(a-\Theta)=\chi_{m}+\sigma_{m}+\left(n-\frac{1}{2}\right) \pi, \quad n \text { an integer }
$$

where

$$
\begin{aligned}
\Theta & =\frac{1}{\pi}(b \ln (d / b)+c \ln (d / c)), \\
\chi_{m} & =\sum_{n=1}^{\infty}\left(\tan ^{-1}\left(\frac{\gamma_{m}^{\prime}}{\gamma_{m n}}\right)-\tan ^{-1}\left(\frac{\gamma_{m}^{\prime}}{\alpha_{m n}}\right)-\tan ^{-1}\left(\frac{\gamma_{m}^{\prime}}{\beta_{m n}}\right)\right),
\end{aligned}
$$

and

$$
\sigma_{m}=\arg \left(1-\sum_{n=1}^{\infty} \frac{A_{m n}}{\gamma_{m n}+\mathrm{i} \gamma_{m}^{\prime}}\right)
$$


where the coefficients $A_{m n}$ are the solutions to the exponentially convergent system of equations

$$
A_{m s}+D_{m s} \sum_{n=1}^{\infty} \frac{A_{m n}}{\gamma_{m s}+\gamma_{m n}}=D_{m s}, \quad s \in \mathbb{N}
$$

with

$$
\begin{aligned}
D_{m s}=2 \gamma_{m s} \mathrm{e}^{2 \gamma_{m s}(\Theta-a)} \frac{\left(\alpha_{m s}-\gamma_{m s}\right)\left(\beta_{m s}-\gamma_{m s}\right)}{\left(\alpha_{m s}+\gamma_{m s}\right)\left(\beta_{m s}+\gamma_{m s}\right)} \\
\quad \times \prod_{\substack{n=1 \\
n \neq s}}^{\infty} \frac{\left(1+\gamma_{m s} / \gamma_{m n}\right)\left(1-\gamma_{m s} / \alpha_{m n}\right)\left(1-\gamma_{m s} / \beta_{m n}\right)}{\left(1+\gamma_{m n}\right)\left(1+\gamma_{m s} / \beta_{m n}\right)\left(1-\gamma_{m s} / \gamma_{m n}\right)}
\end{aligned}
$$

For the case of antisymmetry about $x=0$ we replace the boundary condition (2.1) by

$$
\phi=0 \quad \text { on } x=0, \quad 0<r<d,
$$

and the equivalent condition to $(2.33)$ is

$$
\gamma_{m}^{\prime}(a-\Theta)=\chi_{m}+\sigma_{m}^{\prime}+n \pi, \quad n \text { an integer, }
$$

where $\chi_{m}$ and $\Theta$ are as before and $\sigma_{m}^{\prime}$ is as in (2.36) but with the coefficients $A_{m n}$ coming from the system of equations

$$
A_{m s}-D_{m s} \sum_{n=1}^{\infty} \frac{A_{m n}}{\gamma_{m s}+\gamma_{m n}}=-D_{m s} \quad s \in \mathbb{N} .
$$

\section{Results}

The results in this section are computed with the systems of equations (2.37) and (2.41) truncated to $5 \times 5$ systems. Typical results for the bound-state energies when $m=0$ and 1 are shown in Figure 2 and Figure 3. In both figures the solid lines correspond to modes symmetric about $x=0$ and the dashed lines to modes antisymmetric about $x=0$.

In Figure 2 bound-state energies are plotted against $a / d$ when $b / d=0.5$. As $a / d$ increases more and more modes appear alternately symmetric and antisymmetric about $x=0$ for each value of $m$. The upper and lower cut-offs for the existence of bound states are given by $(2.20)$ and depend on $m$. When $m=0$ the modes appear from the cut-off given by the minimum of $k d=j_{01} / 0.5 \approx 4.810$ and $k d=\nu_{01} d \approx 6.246$, i.e. $k d=2 j_{01}$, and tend to $k d=j_{01} \approx 2.405$ as $a / d$ increases. This minimum value changes however when $m=1$, as the upper cut-off becomes $k d=\nu_{11} d \approx 6.393$ which is less than $k d=2 j_{11} \approx 7.663$. As $a / d$ increases the modes corresponding to $m=1$ tend to $k d=j_{11} \approx 3.832$.

In Figure 3 trapped-mode wavenumbers are plotted against $b / d$ when $a / d=3$. The upper plot corresponds $m=1$ and the lower plot to $m=0$. The dotted lines appearing on both plots are the upper and lower cut-offs for the two different $m$ values and show 


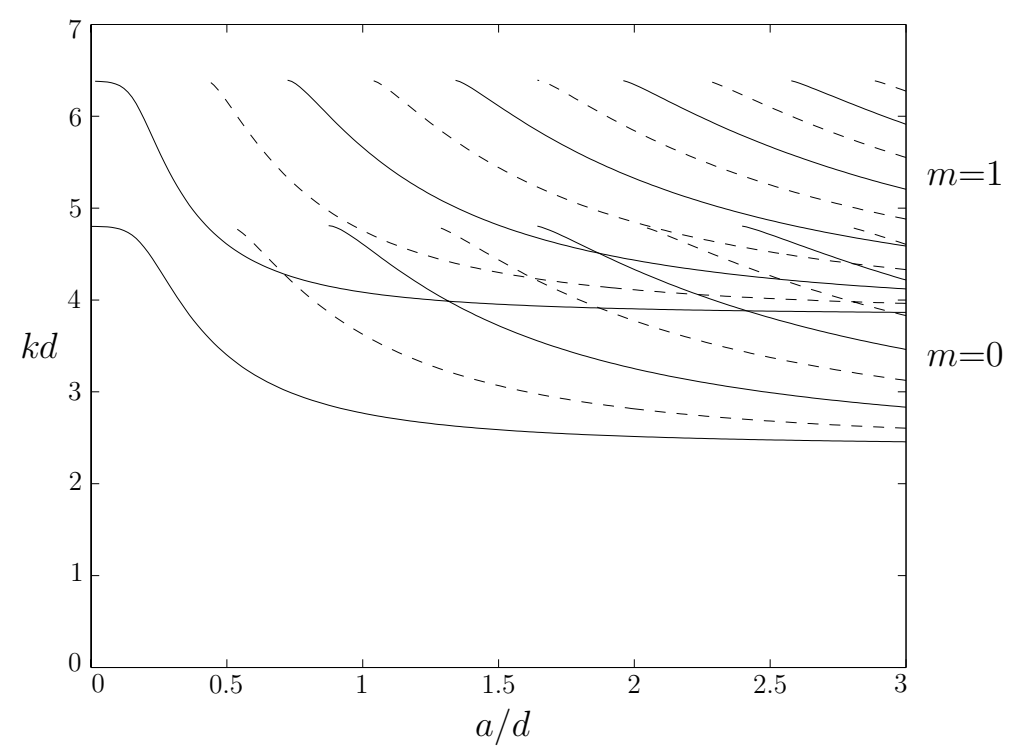

Figure 2: Bound-state energies for modes symmetric (-) and antisymmetric (- -$)$ about $x=0$ plotted against $a / d$ when $b / d=0.5$.

where the frequency ranges lie with respect to each other. When $b / d \rightarrow 0$ the only upper cut-off present for both plots is the one corresponding to $k d=\eta_{m 1}$, whereas when $b / d \rightarrow 1$ the upper cut-off is shown by $k d=d j_{m 1} / b$ for both values of $m$. When $m=0$ the upper cut-off changes from $k d=\eta_{01} d$ to $k d=j_{01} d / b$ when $b / d \approx 0.436$, and the largest number of modes appears at this value. Results for higher values of $m$ show that as $m$ increases the value of $b / d$ when the upper cut-off changes also increases.

\section{Laterally coupled planar waveguides}

We now turn our attention to the case where the two-dimensional laterally coupled waveguide considered in I is rotated about the $y$-axis to produce a three-dimensional waveguide consisting of two planar layers of widths $b$ and $c=d-b$ coupled laterally through a circular hole of radius $a$ in the common boundary. We assume for convenience that $b>d / 2$.

Circular cylindrical polar coordinates $(r, \theta, z)$ are introduced so that the waveguide is axisymmetric about $r=0$ and the planes lie at $z=0, z=b$ and $z=d$. We seek non-trivial solutions $\phi(x, y)$ to

$$
\left(\nabla^{2}+k^{2}\right) \phi=0, \quad 0<z<d, r \geq 0 \text { except on } x=b, r>a,
$$

subject to the boundary conditions

$$
\begin{aligned}
& \phi=0 \text { on } z=0, r \geq 0, \\
& \phi=0 \text { on } z=b, r>a, \\
& \phi=0 \text { on } z=d, r \geq 0,
\end{aligned}
$$



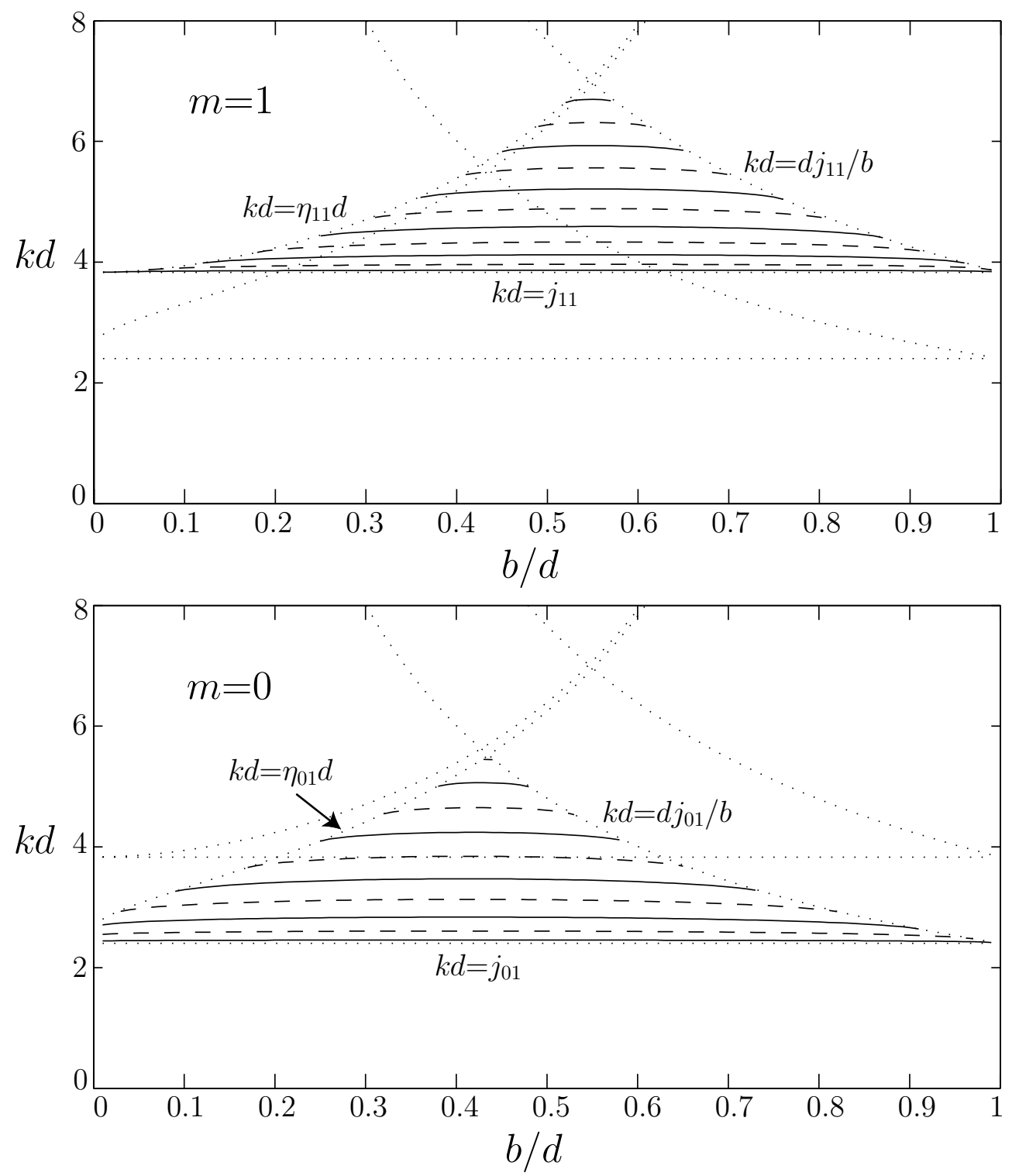

Figure 3: Bound-state energies for modes symmetric $(-)$ and antisymmetric $(--)$ about $x=0$ plotted against $b / d$ when $a / d=3$. 
and a radiation condition specifying that no waves propagate out to infinity,

$$
\phi \rightarrow 0 \text { as } r \rightarrow \infty \text {. }
$$

The guide is divided into three regions. Region $I$ is $\{r, \theta, z: r>a, b<z<d\}$, region $I I$ is $\{r, \theta, z: r>a, 0<z<b\}$ and region $I I I$ is $\{r, \theta, z: r<a, 0<z<d\}$. As the geometry is axisymmetric about $r=0$, we are able to look for a mode with angular variation $\cos m \theta$, $m \in \mathbb{N}_{0}$ and can write $\phi_{i}(r, \theta, z)=\hat{\phi}_{i}(r, z) \cos m \theta,(i=1,2,3)$, in each region and apply the continuity conditions

$$
\hat{\phi}_{i}=\hat{\phi}_{3}, \frac{\partial \hat{\phi}_{i}}{\partial r}=\frac{\partial \hat{\phi}_{3}}{\partial r}, \quad \text { on } L_{i}, i=1,2,
$$

where $L_{1}$ is $r=a, b<z<d, L_{2}$ is $r=a, 0<z<b$ and we write $L_{3}$ for $L_{1} \cup L_{2}$.

Suitable eigenfunction expansions in each region are

$$
\begin{array}{lll}
\hat{\phi}_{1}(r, z) & =\sum_{n=1}^{\infty} U_{m n}^{(1)} \frac{K_{m}\left(\alpha_{n} r\right)}{\alpha_{n} K_{m}^{\prime}\left(\alpha_{n} a\right)} \Psi_{n}^{(1)}(z), & \alpha_{n}=\left(\nu_{n}^{2}-k^{2}\right)^{1 / 2}, \\
\hat{\phi}_{2}(r, z)=\sum_{n=1}^{\infty} U_{m n}^{(2)} \frac{K_{m}\left(\beta_{n} r\right)}{\beta_{n} K_{m}^{\prime}\left(\beta_{n} a\right)} \Psi_{n}^{(2)}(z), & \beta_{n}=\left(\mu_{n}^{2}-k^{2}\right)^{1 / 2}, \\
\hat{\phi}_{3}(r, z)=\sum_{n=0}^{\infty} U_{m n}^{(3)} \frac{I_{m}\left(\gamma_{n} r\right)}{\gamma_{n} I_{m}^{\prime}\left(\gamma_{n} a\right)} \Psi_{n}^{(3)}(z), & \gamma_{n}=\left(\lambda_{n}^{2}-k^{2}\right)^{1 / 2},
\end{array}
$$

where $I_{m}$ and $K_{m}$ are modified Bessel functions and

$$
\begin{array}{lll}
\Psi_{n}^{(1)}(z)=2^{1 / 2} \sin \nu_{n}(d-z), & \nu_{n}=n \pi / c, & n \in \mathbb{N}, \\
\Psi_{n}^{(2)}(z)=2^{1 / 2} \sin \mu_{n}(b-z), & \mu_{n}=n \pi / b, & n \in \mathbb{N}, \\
\Psi_{n}^{(3)}(z)=2^{1 / 2} \sin \lambda_{n}(d-z), & \lambda_{n}=(n+1) \pi / d, & n \in \mathbb{N}_{0},
\end{array}
$$

which satisfy

$$
\frac{1}{\left|L_{i}\right|} \int_{L_{i}} \Psi_{n}^{(i)}(y) \Psi_{m}^{(i)}(y) \mathrm{d} y=\delta_{m n}, \quad i=1,2,3
$$

As in part I we anticipate that a necessary condition for the existence of bound states will be

$$
\pi<k d<\frac{d \pi}{b}
$$

since then $\gamma_{0}$ will be purely imaginary whereas $\alpha_{n}, \beta_{n}$ and $\gamma_{n}, n \in \mathbb{N}$, will all be real and positive. The mode corresponding to $\gamma_{0}$ will therefore be oscillatory in region $I I I$ and all the other modes will decay away from the hole.

We now apply the continuity conditions (3.6), multiply each of the resulting equations by $\Psi_{s}^{(3)}, s \in \mathbb{N}_{0}$ and integrate over $L_{3}$, then eliminate $U_{m s}^{(3)}$ to obtain

$$
\begin{aligned}
\sum_{n=1}^{\infty} U_{m n}^{(1)} d_{n s}\left(\frac{I_{m}\left(\gamma_{n} a\right)}{\gamma_{s} I_{m}^{\prime}\left(\gamma_{s} a\right)}\right. & \left.-\frac{K_{m}\left(\alpha_{n} a\right)}{\alpha_{n} K_{m}^{\prime}\left(\alpha_{n} a\right)}\right) \\
& +\sum_{n=1}^{\infty} U_{m n}^{(2)} e_{n s}\left(\frac{I_{m}\left(\gamma_{n} a\right)}{\gamma_{s} I_{m}^{\prime}\left(\gamma_{s} a\right)}-\frac{K_{m}\left(\beta_{n} a\right)}{\beta_{n} K_{m}^{\prime}\left(\beta_{n} a\right)}\right)=0, \quad s \in \mathbb{N}_{0}
\end{aligned}
$$




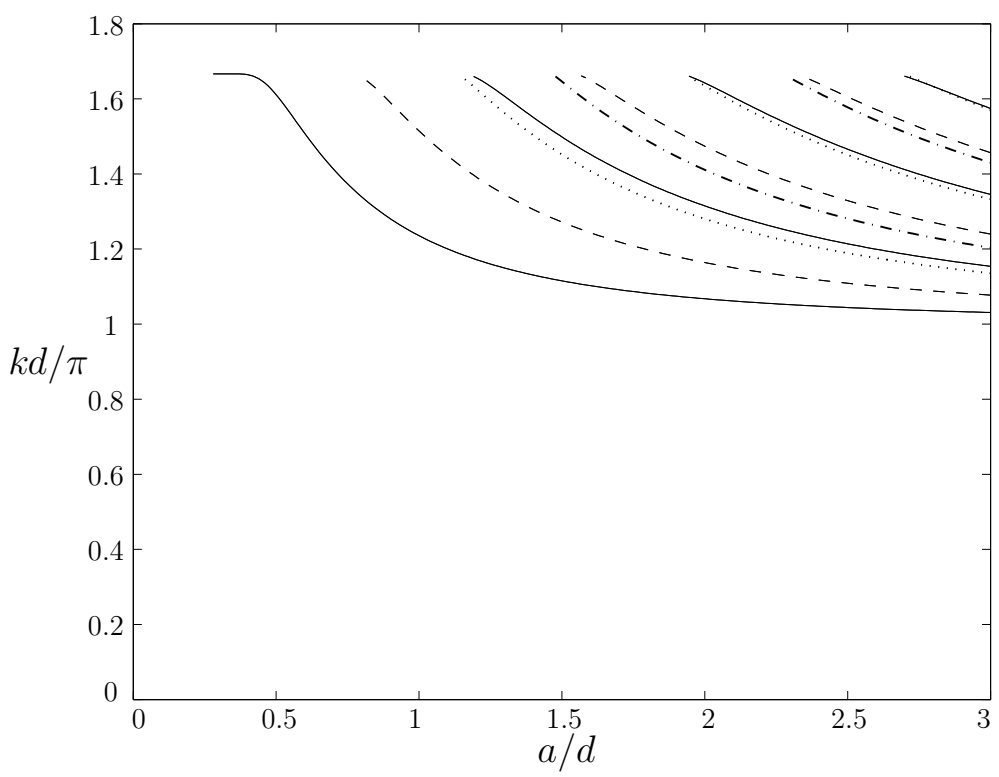

Figure 4: Bound-state energies, $k d / \pi$, plotted against $a / d$ when $b / d=0.6$. The solid lines correspond to $m=0$, the dashed lines to $m=1$, the dotted lines to $m=2$ and the dot-dashed lines to $m=3$.

where $d_{n s}$ and $e_{n s}$ are given by

$$
\begin{array}{lll}
d_{n s}=\frac{1}{d} \int_{L_{1}} \Psi_{n}^{(1)}(y) \Psi_{s}^{(3)}(y) \mathrm{d} y=\frac{2 \nu_{n}(-1)^{n} \sin \lambda_{s} c}{d\left(\gamma_{s}^{2}-\alpha_{n}^{2}\right)}, & s \in \mathbb{N}_{0}, n \in \mathbb{N}, \\
e_{n s}=\frac{1}{d} \int_{L_{2}} \Psi_{n}^{(2)}(y) \Psi_{s}^{(3)}(y) \mathrm{d} y=\frac{2 \mu_{n} \sin \lambda_{s} c}{d\left(\gamma_{s}^{2}-\beta_{n}^{2}\right)}, & s \in \mathbb{N}_{0}, n \in \mathbb{N} .
\end{array}
$$

Equation (3.15) is not amenable to the same residue calculus treatment as we have used in our other examples. Here we solve it directly by truncating the value of $n$ and $s+1$ with a truncation parameter $N$. We then have to find the values of $k d$ for which the determinant of a (real) $2 N \times 2 N$ matrix is zero.

Figure 4 shows a typical set of bound-state energies, $k d / \pi$, plotted against $a / d$ when $b / d=0.6$. The results were computed using a truncation parameter $N=5$. The solid lines correspond to the modes when $m=0$, the dashed lines are when $m=1$, the dotted lines are when $m=2$ and the dot-dashed lines are for $m=3$. As the value of $a / d$ is increased the bound states appear from the upper cut-off $k d=5 \pi / 3$ and tend to the lower cut-off $k d=\pi$. The results suggest that that bound states occur for all $a / d$ except when the hole is small, and that as the value of $m$ is increased the value of $a / d$ below which modes do not exist increases.

However, Exner and Vugalter (1997) used variational techniques to show that for sufficiently small $a / d$ there is just one bound state and that the ground-state eigenvalue, 
$k d$, then satisfies

$$
\frac{d}{b}\left(1-\frac{1}{\pi^{2}} \exp \left(-c_{1} / a^{3}\right)\right)^{\frac{1}{2}} \leq k d \leq \frac{d}{b}\left(1-\frac{1}{\pi^{2}} \exp \left(-c_{2} / a^{3}\right)\right)^{\frac{1}{2}}
$$

for some positive $c_{1}$ and $c_{2}$. It is clear that for small windows, this will produce values of $k d$ which differ from the upper cut-off by an extremely small amount and this explains why we were unable to compute bound-state energies when $a / d$ is less than about 0.25 .

It is fairly simple to extend the work above and consider the case of a pair of planar layers coupled laterally by a number of holes. For simplicity we will consider the case where the two layers have the same width (i.e. $b / d=0.5$ ) and the additional symmetry allows us to consider the symmetric and antisymmetric parts of the solution independently. The antisymmetric part is trivial, and so the problem then reduces to that of a planar guide of width $b$ with Dirichlet boundary conditions on both walls, except for a number of circular discs on one side on which Neumann conditions are applied. The method that we use to analyse multiple circular windows is a standard technique in studying the scattering of waves by arrays of circular cylinders; see, e.g., Linton and McIver (2001) chapter 6.

We consider $P(\geq 1)$ discs and introduce $P+1$ cylindrical polar coordinate systems, so that $(r, \theta, z)$ is centred at the origin and $\left(r_{j}, \theta_{j}, z\right), j=1, \ldots, P$, are centred at the centre of the $j$ th disc, which is assumed to have radius $a_{j}$. The walls of the waveguide are at $z=0$ and $z=b$. The various parameters relating to the relative positions and sizes of the discs are shown in Figure 5. The domain is divided up into $P+1$ parts and we represent the solution in the region above the $j$ th disc by $\phi_{j}^{I}, j=1, \ldots, P$ and the solution exterior to the discs by $\phi^{I I}$. We look for non-trivial solutions to the Helmholtz equation in the guide subject to the boundary conditions

$$
\begin{aligned}
& \phi^{I I}=0 \text { on } z=0, \\
& \phi_{j}^{I}=\phi^{I I}=0 \text { on } z=b, j=1, \ldots, P \text {, } \\
& \frac{\partial \phi_{j}^{I}}{\partial z}=0 \text { on } z=0, r_{j}<a_{j}, j=1, \ldots, P, \\
& \phi^{I I} \rightarrow 0 \text { as } r \rightarrow \infty \text {. }
\end{aligned}
$$

We also need to apply continuity conditions at each region's boundary

$$
\phi_{j}^{I}=\phi^{I I}, \frac{\partial \phi_{j}^{I}}{\partial r_{j}}=\frac{\partial \phi^{I I}}{\partial r_{j}} \quad \text { on } r_{j}=a_{j}, j=1, \ldots, P .
$$

Separation of variables reveals that the appropriate eigenfunction expansions are

$$
\begin{aligned}
\phi_{j}^{I} & =\sum_{m=-\infty}^{\infty} \sum_{n=0}^{\infty} A_{m n}^{j} \frac{I_{m}\left(\alpha_{n} r_{j}\right)}{\alpha_{n} I_{m}^{\prime}\left(\alpha_{n} a_{j}\right)} \Psi_{n}^{(1)}(z) \mathrm{e}^{\mathrm{i} m \theta_{j}}, \quad j=1, \ldots, P, \\
\phi^{I I} & =\sum_{p=1}^{P} \sum_{m=-\infty}^{\infty} \sum_{n=0}^{\infty} B_{m n}^{p} \frac{K_{m}\left(\beta_{n} r_{p}\right)}{\beta_{n} K_{m}^{\prime}\left(\beta_{n} a_{p}\right)} \Psi_{n}^{(2)}(z) \mathrm{e}^{\mathrm{i} m \theta_{p}},
\end{aligned}
$$




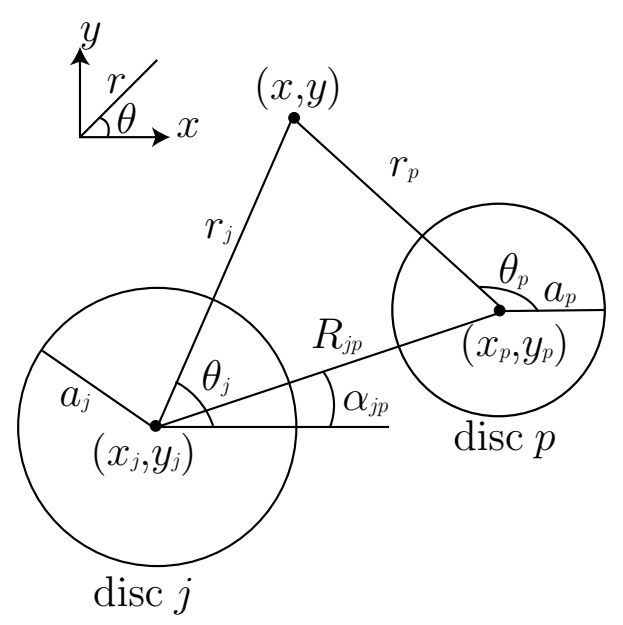

Figure 5: Plan view of two discs.

where $\alpha_{n}=\left(\mu_{n}^{2}-k^{2}\right)^{1 / 2}, \beta_{n}=\left(\nu_{n}^{2}-k^{2}\right)^{1 / 2}$, and

$$
\begin{array}{lll}
\Psi_{n}^{(1)}(z)=2^{1 / 2} \sin \mu_{n}(b-z), & \mu_{n}=\left(n+\frac{1}{2}\right) \pi / b, & n \in \mathbb{N}_{0}, \\
\Psi_{n}^{(2)}(z)=2^{1 / 2} \sin \nu_{n} z, & \nu_{n}=n \pi / b, & n \in \mathbb{N} .
\end{array}
$$

If we restrict the energy so that

$$
\pi<k d<2 \pi
$$

then $\alpha_{0}$ is purely imaginary whereas $\alpha_{n}$ and $\beta_{n}, n \in \mathbb{N}$ will all be real and positive. This restriction allows for wave-like modes local to each disc, with decay as $r$ becomes large.

In order to apply the continuity conditions (3.23) the eigenfunction expansion in region $I I$ must be written in terms of the coordinates $r_{j}$ and $\theta_{j}$. This can be achieved using Graf's addition theorem which shows that provided $r_{j}<R_{j p}$ for all $p$, we can write

$$
\begin{aligned}
\phi^{I I}\left(r_{j}, \theta_{j}, z\right) & =\sum_{m=-\infty}^{\infty} \sum_{n=1}^{\infty} B_{m n}^{j} \frac{K_{m}\left(\beta_{n} r_{j}\right)}{\beta_{n} K_{m}^{\prime}\left(\beta_{n} a_{j}\right)} \Psi_{n}^{(2)}(z) \mathrm{e}^{\mathrm{i} m \theta_{j}} \\
& +\sum_{\substack{p=1 \\
p \neq j}}^{P} \sum_{m=-\infty}^{\infty} \sum_{n=1}^{\infty} \sum_{s=-\infty}^{\infty} B_{m n}^{p} \frac{I_{s}\left(\beta_{n} r_{j}\right)}{\beta_{n} K_{m}^{\prime}\left(\beta_{n} a_{j}\right)} K_{m-s}\left(\beta_{n} R_{j p}\right) \mathrm{e}^{\mathrm{i} s \theta_{p}} \Psi_{n}^{(2)}(z) \mathrm{e}^{\mathrm{i}(m-s) \alpha_{j p}},
\end{aligned}
$$

where $R_{j p}$ is the distance between the centres of discs $j$ and $p$, and $\alpha_{j p}$ is the angle of the centre of disc $p$ from disc $j$, measured as shown in Figure 5.

We can then apply the matching conditions (3.23) and convert the resulting equations into an infinite system by multiplying each by $\Psi_{t}^{(1)}(z) \mathrm{e}^{-\mathrm{i} w \theta_{j}}, t \in \mathbb{N}_{0}, w \in \mathbb{Z}, j=1, \ldots, P$, and integrating over $z$ in $(0, b)$ and $\theta_{j}$ in $(0,2 \pi)$. We obtain, after eliminating $A_{m n}^{j}$,

$$
\sum_{p=1}^{P} \sum_{m=-\infty}^{\infty} \sum_{n=1}^{\infty} B_{m n}^{p} Z_{n m p, t w j}=0, \quad t \in \mathbb{N}_{0}, w \in \mathbb{Z}, j=1, \ldots, P,
$$


where

$$
\begin{gathered}
Z_{n m p, t w j}=\left\{\begin{array}{cc}
d_{t t} X_{t w j} \delta_{m w} \delta_{n t} & p=j, \\
d_{n t} Y_{n m p, t w j} & p \neq j,
\end{array}\right. \\
X_{t w j}=X_{t(-w) j}=\frac{I_{w}\left(\alpha_{t} a_{j}\right)}{\alpha_{t} I_{w}^{\prime}\left(\alpha_{t} a_{j}\right)}-\frac{K_{w}\left(\beta_{t} a_{j}\right)}{\beta_{t} K_{w}^{\prime}\left(\beta_{t} a_{j}\right)}, \\
Y_{n m p, t w j}=K_{m-w}\left(\beta_{n} R_{j p}\right) \mathrm{e}^{\mathrm{i}(m-w) \alpha_{j p}}\left(\frac{I_{w}\left(\alpha_{t} a_{j}\right) I_{w}^{\prime}\left(\beta_{n} a_{j}\right)}{\alpha_{t} I_{w}^{\prime}\left(\alpha_{t} a_{j}\right) K_{m}^{\prime}\left(\beta_{n} a_{j}\right)}-\frac{I_{w}\left(\beta_{n} a_{j}\right)}{\beta_{n} K_{m}^{\prime}\left(\beta_{n} a_{j}\right)}\right)
\end{gathered}
$$

and

$$
d_{n t}=\frac{2}{d} \int_{0}^{d / 2} \Psi_{n}^{(2)}(z) \Psi_{t}^{(1)}(z) \mathrm{d} z=\frac{4 \nu_{n}(-1)^{t}}{d\left(\mu_{t}^{2}-\nu_{n}^{2}\right)} .
$$

\section{Results}

We solve (3.30) by introducing truncation parameters $M$ and $N$ so that $m$ and $w$ vary between $\pm M$, and $n$ and $t+1$ vary between 1 and $N$. Then we write

$$
\begin{aligned}
& \lambda_{1}=n+N(m+M)+N(2 M+1)(p-1), \\
& \lambda_{2}=1+t+N(w+M)+N(2 M+1)(j-1),
\end{aligned}
$$

so that any positive integer $\lambda_{i}$ between 1 and $N(2 M+1) P$ corresponds to a unique triple, $(n, m, p)$ for $i=1$, or $(t, w, j)$ for $i=2$. To find a solution of (3.30) we are required to find frequencies $k d$ so that the determinant of the resulting $N(2 M+1) P \times N(2 M+1) P$ matrix is zero. For the results below truncation parameters $N=5$ and $M=3$ were used.

We consider the case of two circular windows. In this situation the bound-state energies will be independent of the angle between the centres of the two discs $\left(\alpha_{12}\right.$ and $\alpha_{21}$, in Figure 5). That this is the case can be demonstrated as follows. The matrix $Z$ has a $2 \times 2$ block structure and if we multiply the rows in the blocks corresponding to $j=1$ by $\exp \left(-\mathrm{i} m \alpha_{12}\right)$, the rows in the blocks corresponding to $j=2$ by $\exp \left(-\mathrm{i} m \alpha_{21}\right)$, the columns in the blocks corresponding to $p=1$ by $\exp \left(\mathrm{i} w \alpha_{21}\right)$, and the columns in the blocks corresponding to $p=2$ by $\exp \left(\mathrm{i} w \alpha_{12}\right)$, the resulting matrix is independent of the angles $\alpha_{12}$ and $\alpha_{21}$ and its determinant is a non-zero multiple of the determinant of $Z$ and so will vanish for the same values of $k d$.

Figure 6 shows the bound-state energies, $k d / \pi$ plotted against non-dimensional separation $R / d$ for two identical windows with radius $a=d$. The dotted lines are the trapped-mode wavenumbers from the single window case when $a / d=1$ and are labelled with the appropriate values of $m$. They represent the limits for the energies as $R / d \rightarrow \infty$. The dashed line is the upper cut-off $k d=2 \pi$. All possible modes appear in the figure. However, if we took a larger value of $a / d$ we would have to use a larger truncation parameter $M$ to find all the modes.

When the two discs are touching, i.e. when $R / d=2$, there are fourteen bound states. There are two modes around each dotted line corresponding to $m=0$ and four modes 

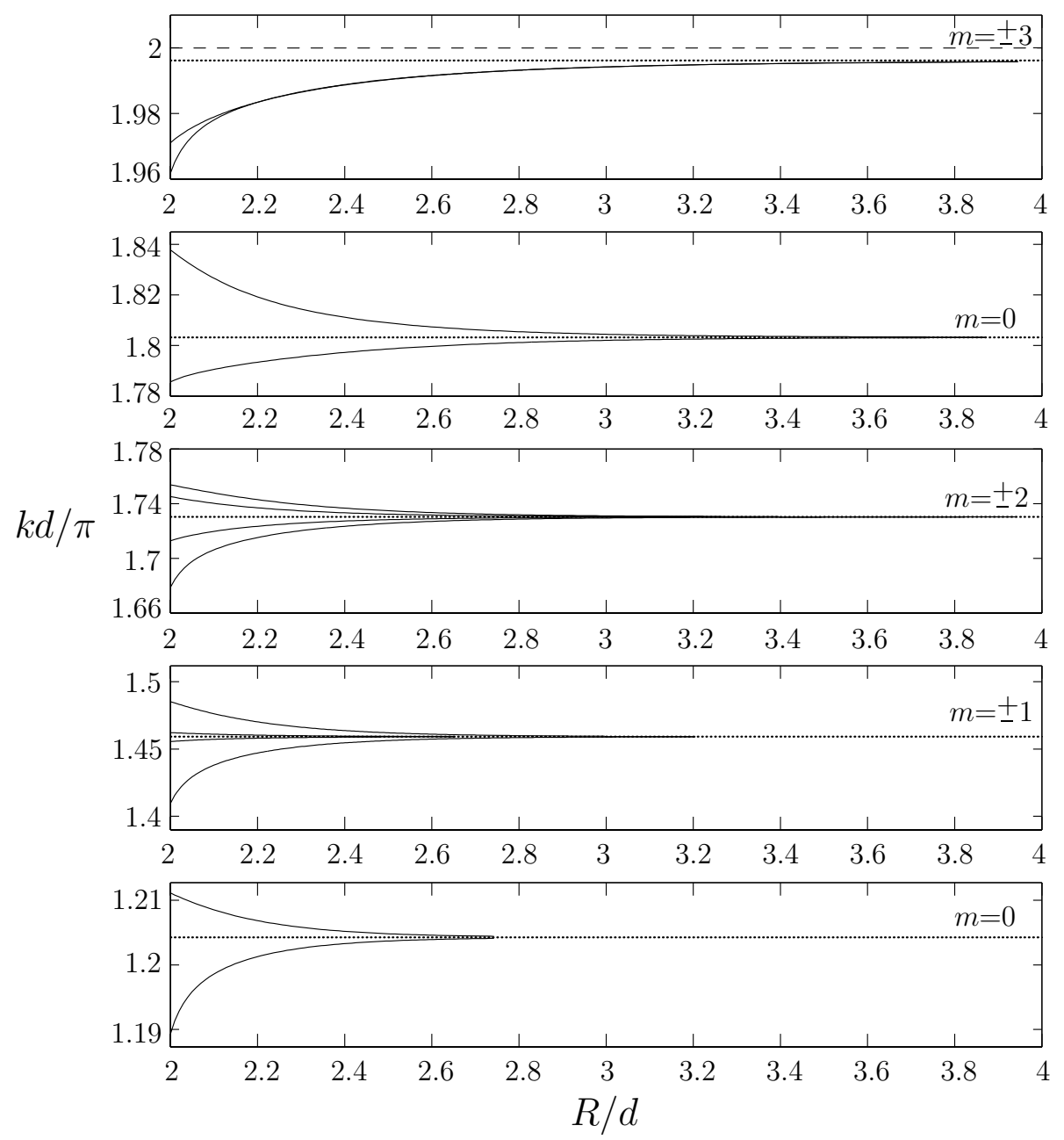

Figure 6: Bound-state energies, $k d / \pi$, plotted against $R / d$ for the case of two identical windows with radius $a=d$. The dotted lines correspond to the bound states from the single disc case with $a / d=1$ and are labelled with the appropriate values of $m$. The dashed line corresponds to the upper cut-off. 
around the other dotted lines (two for the positive value of $m$ and two for the negative value of $m$ ). The exception occurs when $m= \pm 3$ as this mode is close to the upper cut-off and so only a single mode appears below the dotted line for each $m$. As the value of $R / d$ is increased, which means that the discs are moved apart, each pair of modes converges towards the limiting case.

Figure 7 shows the bound-state energies, $k d / \pi$ plotted against $R / d$ for two circular windows for which $a / d=1$ and $a / d=0.75$, respectively. The dotted lines are the bound-state energies from the single window cases when $a / d=1$ and $a / d=0.75$ and are labelled with the appropriate values of $m$ and again represent the limits for the energies as $R / d \rightarrow \infty$. The dashed lines are the upper and lower cut-off's. All possible modes appear in the figure. Between the two cut-off's we find that there are five modes from the single window case with $a / d=1$ and three modes from the single window case when $a / d=0.75$. When the two windows are touching, i.e. when $R / d=1.75$, the boundstate energies are very different from the single window values, but as the separation is increased, the energies quickly approach the single window values. Similar types of results were found by Evans and Porter (1997) who computed trapped modes in the vicinity of multiple cylinders in a channel.

\section{Conclusion}

We have computed bound-state energies below the first cut-off in two three-dimensional coupled waveguides, each obtained from the two-dimensional configuration considered in I by rotating the geometry about a different axis.

First, we have studied bound states in a waveguide consisting of two concentric circular cylindrical waveguides coupled by a finite length gap along the axis of the inner cylinder. The same residue calculus method which was used in I was used to compute bound-state energies below the first cut-off for wave propagation down the guide. We were able to look for modes with a given angular variation $\cos m \theta, m \in \mathbb{N}_{0}$, and the bound states found are either symmetric and antisymmetric about the line of symmetry perpendicular to the axis of the cylinder. The available energy band for bound states is dependent on the values of $m$ and the ratio of the two radii. The results show that bound states occur for any value of the gap length and ratio of radii, and the energies increase as $m$ increases.

Secondly, we have considered the problem in which a pair of planar layers are coupled laterally by a circular hole. The sophisticated residue calculus theory is not available in this case, but we were able to compute bound states by searching for the zeros of the determinant of a matrix found from a mode-matching approach. For this problem we can again consider a given angular variation $\cos m \theta, m \in \mathbb{N}_{0}$, and find modes in each case.

We then extended the theory to cover the case of a number of circular windows connecting two planar guides of equal width and presented results for two circles; both when they have the same radius and when they do not. In both situations we find that when 


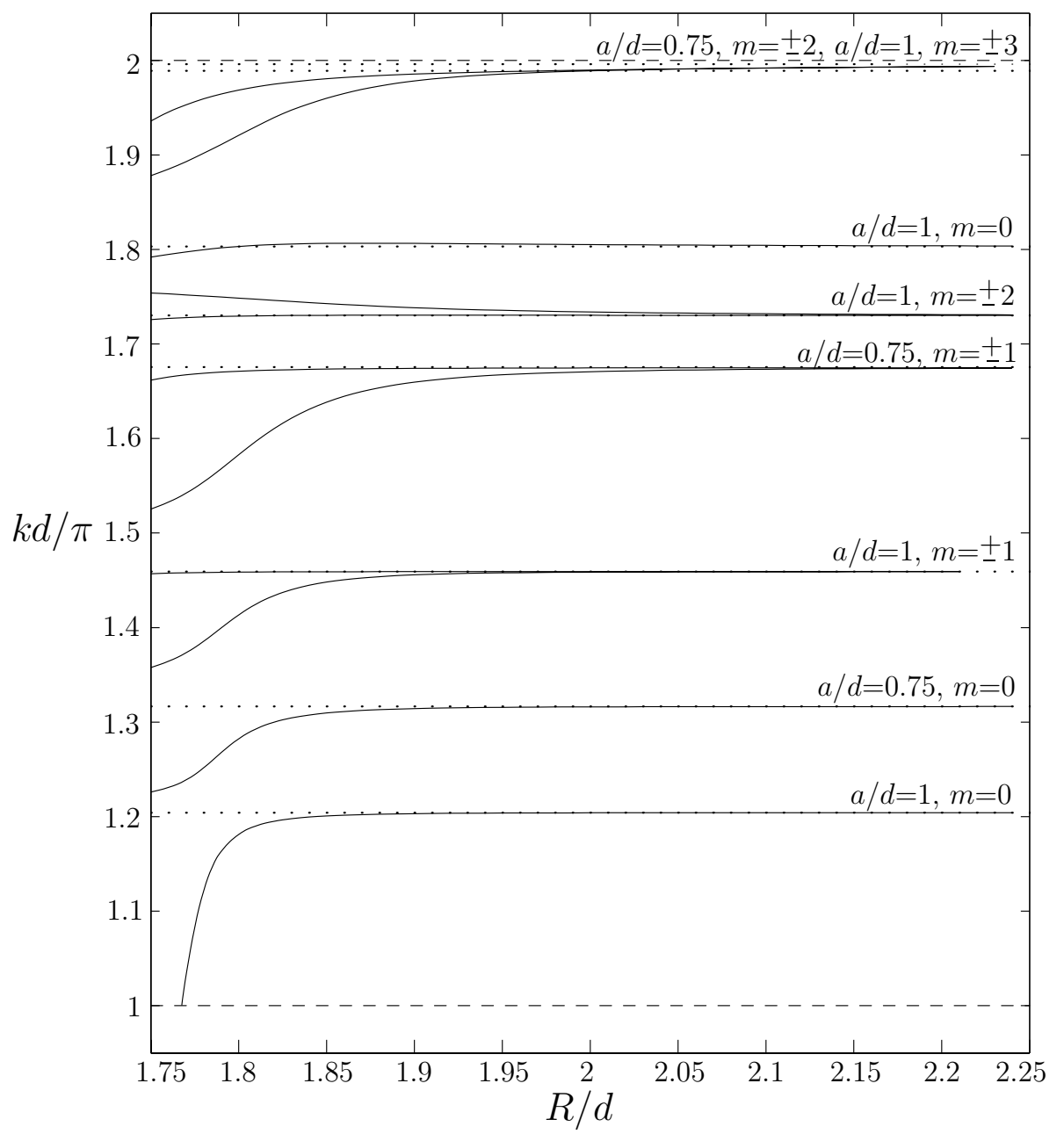

Figure 7: Bound-state energies, $k d / \pi$, plotted against $R / d$ for the case of two windows of radii $a / d=1$ and $a / d=0.75$. The dotted lines correspond to the bound states from the single window case with $a / d=1$ and $a / d=0.75$ and are labelled with the appropriate values of $m$. The dashed lines correspond to the upper and lower cut-off's. 
the windows are far apart the bound states are equivalent to those occuring in the single window cases, but that the energies vary considerably as the windows are moved closer together.

\section{References}

Abramowitz, M. and I. A. Stegun (1965). Handbook of Mathematical Functions. Dover Publications, New York.

Davies, E. B. and L. Parnovski (1998). Trapped modes in acoustic waveguides. Q. J. Mech. Appl. Math. 51, 477-492.

Evans, D. V. and C. M. Linton (1994). Acoustic resonance in ducts. J. Sound Vib. 173(1), 85-94.

Evans, D. V. and R. Porter (1997). Trapped modes about multiple cylinders in a channel. J. Fluid Mech. 339, 331-356.

Exner, P. and S. A. Vugalter (1997). Bound-state asymptotic estimates for windowcoupled Dirichlet strips and layers. J. Phys. A 30, 7863-7878.

Groves, M. D. (1998). Examples of embedded eigenvalues for problems in acoustic waveguides. Math. Meth. in the Appl. Sci. 21, 479-488.

Jones, D. S. (1986). Acoustic and Electromagnetic Waves. Clarendon Press, Oxford.

Linton, C. M. and M. McIver (1998). Trapped modes in cylindrical guides. Q. J. Mech. Appl. Math. 51, 389-412.

Linton, C. M. and P. McIver (2001). Handbook of Mathematical Techniques for Wave/Structure Interactions. Chapman \& Hall/CRC, Boca Raton.

Mittra, R. and S. W. Lee (1971). Analytical Techniques in the Theory of Guided Waves. Macmillan, New York.

Popov, I. Y. (2002). Asymptotics of bound states and bands for laterally coupled waveguides and layers. J. Math. Phys. 43, 215-234.

Ursell, F. (1991). Trapped modes in a circular cylindrical acoustic waveguide. Proc. Roy. Soc. Lond., A 435, 575-589.

Witsch, K. J. (1990). Examples of embedded eigenvalues for the Dirichlet-Laplacian in domains with infinite boundaries. Math. Meth. in the Appl. Sci. 12, 177-182. 